\title{
BJO
}

\section{Simultaneous spectral domain OCT and fundus autofluorescence imaging of the macula and microperimetric correspondence after successful repair of rhegmatogenous retinal detachment}

Wico W Lai, Gloria Y O Leung, Catherine W S Chan, et al.

Br J Ophthalmol 2010 94: 311-318 originally published online October 12, 2009

doi: $10.1136 /$ bjo.2009.163584

Updated information and services can be found at:

http://bjo.bmj.com/content/94/3/311.full.html

\section{These include:}

References This article cites 24 articles, 6 of which can be accessed free at: http://bjo.bmj.com/content/94/3/311.full.html\#ref-list-1

Email alerting Receive free email alerts when new articles cite this article. Sign up in the service box at the top right corner of the online article.

Notes

To request permissions go to:

http://group.bmj.com/group/rights-licensing/permissions

To order reprints go to:

http://journals.bmj.com/cgi/reprintform

To subscribe to BMJ go to:

http://journals.bmj.com/cgi/ep 


\title{
Simultaneous spectral domain OCT and fundus autofluorescence imaging of the macula and microperimetric correspondence after successful repair of rhegmatogenous retinal detachment
}

\author{
Wico W Lai, Gloria Y 0 Leung, Catherine W S Chan, lan Y L Yeung, David Wong
}

From the Eye Institute, and the Research Center of Heart, Brain, Hormone and Healthy Ageing, The University of Hong Kong, Pokfulam, Hong Kong

\section{Correspondence to}

Dr Wico W Lai, Eye Institute, The University of Hong Kong, Room 301, Block B, Cyberport 4, 100 Cyberport Road, Hong Kong; wicolai@hku.hk

Accepted 23 August 2009

\section{ABSTRACT}

Background/aims To evaluate the ultrastructural changes in the macula after successful repair of rhegmatogenous retinal detachment (RRD) using simultaneous spectral domain optical coherence tomography (sdOCT) and fundus autofluorescence (FAF) imaging and to perform functional correlations with microperimetry.

Methods Simultaneous sdOCT and FAF imaging were performed using a combined sdOCT-confocal scanning laser ophthalmoscope (cSLO) system. Microperimetry was performed in a subgroup of these patients.

Results 17 patients with macular-on RRD and 20 with macular-off RRD were recruited. sdOCT images revealed disruption of the external limiting membrane, inner segment/outer segment junction or the Verhoeff membrane in 16 eyes (43.2\%). The presence of any or more of these disruptions was significantly associated with a poor postoperative best-corrected visual acuity (BCVA) $(p<0.001)$. Patients with abnormal FAF were also associated with poor postoperative BCVA $(p<0.001)$. However, there was a generally poor correspondence between the localisation of ultrastructural abnormalities as detected by sdOCT and FAF changes. Areas of decreased microperimetric sensitivity corresponded well with the ultrastructural changes and abnormal FAF.

Conclusions FAF changes may lag behind ultrastructural changes in the OCT, some of which may reverse with the passage of time. The present study demonstrates the ultrastructural changes assessed with simultaneous FAF and SdOCT and their relation with visual outcome.

\section{INTRODUCTION}

Incomplete visual recovery following successful repair of rhegmatogenous retinal detachment (RRD) can often occur in clinically normal-looking retina. Cystoid macular oedema (CMO), epiretinal membranes (ERM) and retinal folds have been reported to contribute to the delayed or incomplete visual recovery, but they account for only a minority of cases. ${ }^{12}$ The advent of optical coherence tomography (OCT) has allowed us to detect ultrastructural changes which may not be clinically observable. Persistent subretinal fluid (SRF), CMO and intraretinal separation have been detected. ${ }^{2-6}$ Spectral (Fourier) domain OCT (sdOCT) allows high-resolution and rapid imaging with reduced motion artefact, with images reaching histological details. ${ }^{7}$ sdOCT has detected disruption of the photoreceptor inner and outer segment (IS/OS) junction in macular-off RRD both preoperatively ${ }^{8}$ and postoperatively, ${ }^{9}{ }^{10}$ suggesting photoreceptor damage.
Fundus autofluorescence (FAF) is derived predominantly from lipofuscin in the retinal pigment epithelium (RPE). ${ }^{11}$ FAF imaging by the confocal scanning laser ophthalmoscope (cSLO) has been used in the evaluation of retinal conditions and the effects of treatment. ${ }^{12-14}$ In addition to diseases that affect the RPE, those that result in changes in the outer retina and subretinal space may also lead to FAF abnormalities. ${ }^{15}$

In this study, we performed a cross-sectional study using simultaneous sdOCT and FAF imaging in eyes that had undergone repair of both macularon and macular-off RRD. The advantage of simultaneous imaging is that we can correlate and colocalise in a point-to-point manner ultrastructural changes on sdOCT with FAF changes. It is already known that visual function can be related to sdOCT changes in outer retina. ${ }^{8} 10$ Our hypothesis is that there may also be an association between FAF changes and morphological and visual outcome. We tested this hypothesis not only by using best-corrected visual acuity (BCVA) but also by mapping retinal sensitivity using microperimetry. Our hypothesis is that there may also be an association between FAF changes and morphological and visual outcome.

\section{METHODS}

The medical records of patients who presented with primary $\mathrm{RRD}$ to the Eye Institute, The University of Hong Kong, were reviewed. Those patients who had a successful repair of the RRD after a single surgery for at least 6 months were recruited. Patients with media opacities and macular pathologies (eg, diabetic macular oedema) were excluded. The study was approved by our Institution Review Board. Written informed consent was obtained prior to study participation.

The following demographic data were collected: patient's age, sex, preoperative Snellen BCVA, duration of symptoms preoperatively, extent of $\mathrm{RRD}$ and surgical procedures performed. Eligible patients underwent postoperative BCVA testing, anterior segment and dilated fundus examinations.

Recruited subjects underwent simultaneous sdOCT and FAF imaging using the Spectralis HRA +OCT (Heidelberg Engineering, Heidelberg, Germany). This device combines high-resolution sdOCTwith a cSLO and provides exact point-point correlation of ultrastructural changes with FAF. ${ }^{16}$ The combination of cSLO and sdOCT allows for real-time tracking of eye movements and averaging 
of cSLO images and OCT scans, thereby reducing motion artefacts.

Seventeen patients underwent microperimetry using the Micro Perimeter 1 (MP-1, Nidek Technologies, Padua, Italy). MP-1 allows correlation between functional defects and macular pathology. It is capable of mapping out microscotomas present in the central visual field. These points are then registered onto corresponding fundus photographs, thereby allowing localisation of the functional defects to macular pathologies. ${ }^{17}$ A customised pattern of 45 stimuli was set, covering the central $20^{\circ}$ and 17 stimuli for the central $2^{\circ}$. Complete randomisation of the stimuli with reliability test was presented. The classification of fixation characteristics is made automatically by the microperimeter software.

To correlate functional deficits with FAF and sdOCT findings, MP-1 and FAF images were superimposed using the MP-1's registration software. FAF images were imported to the MP-1, and both images were shown in the output screen. A total of four landmarks on each of the images were selected, and registration between the images was performed.

For statistical analysis, patients were arbitrarily divided into two groups based on their postoperative BCVA: group A with $B C V A>20 / 60$ (0.52 logMAR); group $B$ with $B C V A \leq 20 / 60$. Descriptive statistics were used to determine the distribution of demographic and clinical characteristics. The differences in gender, preoperative macular status, ultrastructural changes in the outer retina and FAF findings between groups A and B were compared using the $\chi^{2}$ test. Other continuous variables, including age, preoperative BCVA and postoperative MP-1, were compared using a non-parametric Mann-Whitney $U$ test. Spearman $\rho$ was calculated to determine the correlations between preoperative BCVA and postoperative BCVA and postoperative BCVA and MP-1. All analyses were conducted at the 5\% significance level, using the Statistical Package for Social Sciences (SPSS) version 16.0 (SPSS, Chicago).

\section{RESULTS}

\section{Demographics}

Thirty-seven eyes of $37 \mathrm{RRD}$ patients were included. Of these, 17 patients had macular-on RRD, and 20 had macular-off RRD. There were 19 patients in group A and 18 in group B. The mean preoperative logMAR BCVA in groups A and B were 0.41 (range $=0.10-2.30$ ) and 0.96 (range $=0.10$ to light perception), respectively. The mean postoperative logMAR BCVA improved to 0.21 in group A. However, there was no improvement in BCVA in group $\mathrm{B}$. The time between symptom development and surgery was $5.03 \pm 7.23$ days. Ten patients (27\%) underwent a sclera buckling (SB) procedure, and 24 (65\%) underwent a pars plana vitrectomy (PPV). The rest of the patients had either a combination of SB and PPV (3\%) or pneumatic retinopexy $(5 \%)$. The mean time between surgery and sdOCT examination was $7.62 \pm 3.24$ months. Preoperatively, only macular status $(\mathrm{p}=0.001)$ and logMAR BCVA $(\mathrm{p}<0.001)$ were significantly different between groups $\mathrm{A}$ and $\mathrm{B}$ (table 1 ).

\section{sdOCT changes}

sdOCT revealed ultrastructural changes in 23 eyes (62.2\%). SRF was seen in five eyes (13.5\%), CMO in four eyes $(10.8 \%)$, isolated ERM in three eyes (8.1\%) and combined ERM and macular folds (MF) in two (5.4\%) eyes. Disruptions of the external limiting membrane and/or inner segment/outer segment junction and/or the Verhoeff membrane were noted in $16(43.2 \%)$ patients. In group A, there was no disruption of the ELM or IS/OS in any of the patients (figure 1B). Only one patient $(5.3 \%)$ had disruption of the Verhoeff membrane (VM), ${ }^{7}$
Table 1 Demographic and clinical characteristics of patients after repair of rhegmatogenous retinal detachment

\begin{tabular}{ll}
\hline No of patients & 37 \\
No of eyes & 37 \\
Age (years; mean \pm SD) & $51.86 \pm 16.63$ \\
Gender (no/\%) & \\
$\quad$ Male & $25(68)$ \\
$\quad$ Female & $12(32)$ \\
Time between symptoms and surgery & $5.03 \pm 7.23$ \\
(days; mean \pm SD) & \\
Preoperative BCVA (mean; range) & \\
$\quad$ Snellen & 0.25 (LP to 0.80$)$ \\
$\quad$ logMAR & 0.60 (LP to 0.10) \\
Macular status (no/\%) & \\
$\quad$ On & $17(46)$ \\
$\quad$ Off & $20(54)$ \\
Surgery performed (no/\%) & \\
SB & $10(27)$ \\
PPV & $24(65)$ \\
$\quad$ SB + PPV & $1(3)$ \\
PR & $2(5)$ \\
Postoperative BCVA (mean; range) & \\
Snellen & 0.37 (HM to 1.00) \\
LogMAR & $0.43(2.30$ to 0.00) \\
Time between surgery and OCT (months; & $7.62 \pm 3.24$ \\
mean + SD) &
\end{tabular}

mean \pm SD)

BCVA, best-corrected visual acuity; HM, hand movement; logMAR, logarithm of the minimal angle of resolution; LP, light perception; OCT, optical coherence tomography; PPV, pars plana vitrectomy; PR, pneumatic retinopexy; SB, scleral buckle.

the backreflection located anterior to the RPE and posterior to the OS. In group B, 15 patients (83.3\%) had ultrastructural abnormalities. Eleven patients (61.1\%) had ELM disruption, 15 (83.3\%) had IS/OS disruption, and 14 (77.8\%) had VM disruption. Of these patients, one eye (5.6\%) had isolated IS/OS disruption, three eyes (16.7\%) had combined IS/OS junction and VM disruption, and 11 eyes (61.1\%) had disruption of all three layers. The percentage of patients with disruption of one or more outer retinal layers was significantly different between groups $A$ and $B$ $(p<0.001)$. A subgroup analysis was also performed between patients with macular-on and those with macular-off RRD to show the effects of the RRD on the morphological outcomes in the macula. The differences in the disruptions of the ELM, IS/OS and VM were significantly different between the two groups $(\mathrm{p}=0.002)$ (table 2).

\section{FAF changes}

Abnormal FAF was observed in 21 (56.8\%) of 37 patients. They occurred in three group A patients (15.8\%) and in all group B patients. The abnormal FAF was patchy, curvilinear (figure 1D) or isolated in appearance (figure 1I). In group A, one patient had $\mathrm{CMO}$, and two had SRF. Both exhibited increased FAF.

\section{Correspondence of sdOCT and FAF changes}

In group $\mathrm{A}$, there was one patient with isolated VM disruption. The FAF was normal in this patient and in the rest of the patients in the group. In group $B$, all patients had abnormal FAF. Two patients with ERM and two with combined ERM and macular folds had decreased FAF. There was one patient with isolated IS/OS disruption who had a corresponding increase in FAF. The remaining three patients all had disruptions of both IS/ $O S$ and VM. Of these, one had curvilinear areas of increased FAF (figure 1D,E), one had patchy areas of increased FAF, and one had decreased FAF. Another 11 patients had disruptions of all three layers. Of these, six patients had areas of increased FAF (figure 1I, $J$ ), and five had decreased FAF. Disruptions in any of the outer 

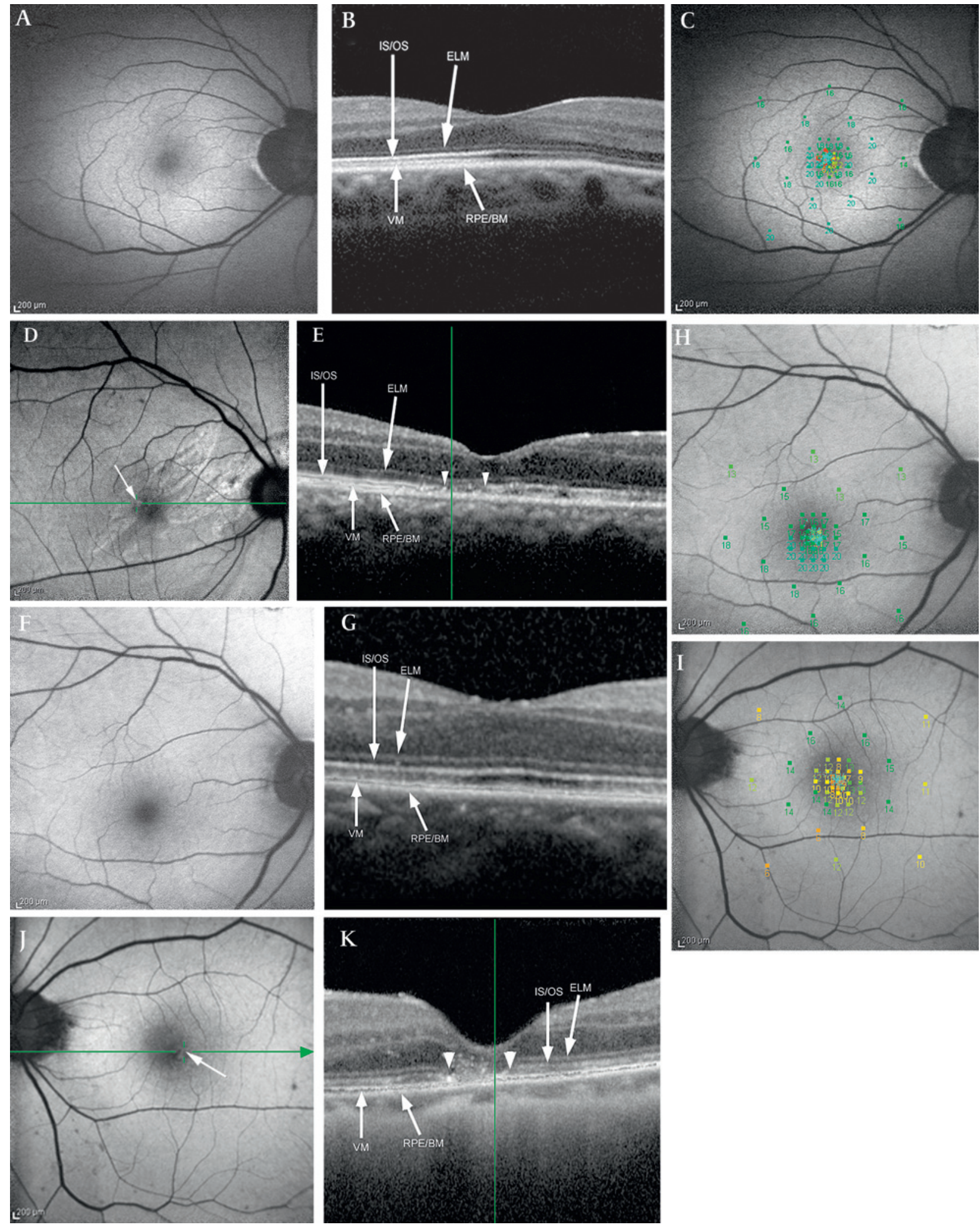

Figure 1 Simultaneous postoperative FAF imaging and sdOCT, and MP-1 analysis. $(A-C)$ Patient 1. Preoperative $B C V A=6 / 12 ;$ postoperative $B C V A=6 / 15$. (A) Normal FAF. (B) All layers of the retina are intact on sdOCT. (C) MP-1 shows good central fixation and normal mean sensitivity. (D-E) Patient 22. Preoperative $B C V A=6 / 15$; postoperative $B C V A=6 / 60$. (D) Curvilinear hyperfluorescence on $F A F$ imaging (arrow). (E) sdOCT shows disruptions of the IS/OS and VM (arrowheads). The boundaries of the increase FAF did not colocalise exactly with those of the disruptions (green vertical line). (F-H) Patient 27. Preoperative $B C V A=6 / 120$; postoperative $B C V A=6 / 12$. (F, G) FAF and sdOCT were normal. (H) MP-1 showed normal sensitivity in the macula. (I-K) Patient 34. Preoperative $\mathrm{BCVA}=\mathrm{HM}$; postoperative BCVA=6/18. (I) Increase FAF (arrow). (J) Disruptions of the ELM, IS/OS and VM are noted on sdOCT (arrowheads). The boundaries of the increased FAF did not colocalise exactly with those of the disruptions (green vertical line). (K) MP-1 revealed decreased sensitivity in the area of increased FAF and disruptions. BCVA, best-corrected visual acuity; ELM, external limiting membrane; FAF, fundus autofluorescence; HM, hand movement; IS/OS, photoreceptor inner and outer segment junction; MP-1, microperimetry; sdOCT, spectral domain optical coherence tomography; VM, Verhoeff membrane. 
Table 2 Clinical and spectral domain optical coherence tomographic characteristics between rhegmatogenous retinal detachment patients with good and poor postoperative best-corrected visual acuity and different macular status

\begin{tabular}{|c|c|c|c|c|c|c|}
\hline & \multicolumn{2}{|l|}{ Postoperative BCVA } & \multirow[b]{2}{*}{$\begin{array}{l}p \\
\text { Value* }\end{array}$} & \multicolumn{2}{|l|}{ Macular status } & \multirow[b]{2}{*}{$\begin{array}{l}\text { p } \\
\text { Value* }\end{array}$} \\
\hline & $\begin{array}{l}\text { Group } A \\
\text { BCVA>20/60 (0.52 } \\
\log M A R)(n=19)\end{array}$ & $\begin{array}{l}\text { Group B } \\
\text { BCVA } \leq 20 / 60 \\
(n=18)\end{array}$ & & $\begin{array}{l}\text { Macular on } \\
(n=17)\end{array}$ & $\begin{array}{l}\text { Macular off } \\
(n=20)\end{array}$ & \\
\hline Gender & & & 0.414 & & & 0.717 \\
\hline Male & $14(73.7)$ & $11(61.1)$ & & $12(70.6)$ & $13(65)$ & \\
\hline Female & $5(26.3)$ & 7 (38.9) & & 5 (29.4) & 7 (35) & \\
\hline Macula detached & $5(26.3)$ & $15(83.3)$ & & & & \\
\hline Preoperative BCVA (mean; range) & & & $<0.001$ & & & $<0.001$ \\
\hline Snellen & 0.39 (HM to 0.80$)$ & 0.11 (LP to 0.80$)$ & & $0.52(0.30$ to 0.80$)$ & 0.03 (LP to 0.30$)$ & \\
\hline LogMAR & $0.41(2.30$ to 0.10$)$ & 0.96 (LP to 0.10$)$ & & $0.28(0.52$ to 0.10$)$ & 1.52 (LP to 0.52$)$ & \\
\hline Postoperative BCVA (mean; range) & & & $<0.001$ & & & $<0.001$ \\
\hline Snellen & $0.61(0.40$ to 1.00$)$ & 0.11 (HM to 0.30 ) & & $0.56(0.10$ to 1.00$)$ & 0.21 (HM to 0.70 ) & \\
\hline IS/OS disruption (no/\%) & & & $<0.001$ & & & $<0.001$ \\
\hline Yes & $0(0)$ & $15(83.3)$ & & $1(5.9)$ & $14(70)$ & \\
\hline No & $19(100)$ & $3(16.7)$ & & $16(94.1)$ & $6(30)$ & \\
\hline VM disruption (no/\%) & & & $<0.001$ & & & 0.001 \\
\hline Yes & $1(5.3)$ & $14(77.8)$ & & $2(11.8)$ & $13(65)$ & \\
\hline No & 18 (94.7) & $4(22.2)$ & & 15 (88.2) & 7 (35) & \\
\hline Outer retina status (no/\%) & & & $<0.001$ & & & 0.002 \\
\hline $\mathrm{ELM}(+) / \mathrm{IS} / \mathrm{OS}(+) / \mathrm{VM}(+)$ & $18(94.7)$ & $3(16.7)$ & & $15(88.2)$ & $6(30)$ & \\
\hline $\mathrm{ELM}(+) / \mathrm{IS} / \mathrm{OS}(+) / \mathrm{VM}(-)$ & $1(5.3)$ & $0(0)$ & & $1(5.9)$ & $0(0)$ & \\
\hline $\mathrm{ELM}(+) / \mathrm{IS} / \mathrm{OS}(-) / \mathrm{VM}(+)$ & $0(0)$ & $1(5.5)$ & & $0(0)$ & $1(5)$ & \\
\hline $\mathrm{ELM}(+) / \mathrm{IS} / \mathrm{OS}(-) / \mathrm{NM}(-)$ & $0(0)$ & $3(16.7)$ & & $1(5.9)$ & $2(10)$ & \\
\hline $\mathrm{ELM}(+) / / \mathrm{S} / \mathrm{OS}(-) / \mathrm{VM}(+) / \mathrm{FAF}(\mathrm{A})$ & $0(0)$ & $1(5.5)$ & & $0(0)$ & $1(5)$ & \\
\hline $\mathrm{ELM}(+) / / \mathrm{S} / \mathrm{OS}(-) / \mathrm{NM}(-) / \mathrm{FAF}(\mathrm{A})$ & $0(0)$ & $3(16.7)$ & & $1(5.9)$ & $2(10)$ & \\
\hline ELM(-)/IS/OS(-)/VM(-)/FAF(A) & $0(0)$ & $11(61.1)$ & & $0(0)$ & $11(55)$ & \\
\hline $\operatorname{SRF}(\mathrm{no} / \%)$ & $2(10.5)$ & $3(16.7)$ & 0.271 & $2(11.8)$ & $3(15)$ & 0.759 \\
\hline CMO (no/\%) & $1(5.3)$ & $3(16.7)$ & 0.150 & $1(5.9)$ & $3(15)$ & 0.446 \\
\hline ERM (no/\%) & $1(5.3)$ & $4(22.2)$ & 0.076 & $2(11.8)$ & $3(15)$ & 0.825 \\
\hline ERM and macular fold (no/\%) & $0(0)$ & $2(11.1)$ & 0.081 & $1(5.9)$ & $1(5)$ & 0.902 \\
\hline Cataract (no/\%) & $1(5.3)$ & $0(0)$ & 0.455 & $1(5.9)$ & $0(0)$ & 0.286 \\
\hline
\end{tabular}

*Probabilities are from the $\chi^{2}$ test (categorical variables) and Mann-Whitney $\mathrm{U}$ test (continuous variables) contrasting rhegmatogenous retinal detachment patients with good and poor postoperative best-corrected visual acuity and different macular status.

'-,' photoreceptor disruption; '+,' no disruption; A, abnormal; BCVA, best-corrected visual acuity; CMO, cystoid macular oedema; ELM, external limiting membrane; ERM, epiretinal membrane; FAF, fundus autofluorescence; HM, hand movement; IS/OS, photoreceptor inner and outer segment junction; logMAR, logarithm of the minimal angle of resolution; LP, light perception; N, normal; SRF, subretinal fluid; VM, Verhoeff membrane.

retinal layers were significantly associated with abnormal FAF $(\mathrm{p}<0.001)$. However, by moving the curser along the line of cut with the sdOCT, we found that the boundaries of FAF changes did not always correspond exactly with the boundaries of the ultrastructural changes (figure 1D,E,I,J), that is, the ultrastructural and FAF changes did not exactly colocalise.

\section{Correlation of sdOCT and FAF changes with visual acuity and macula status}

Patients with normal FAF were associated with good VA postoperatively $(p<0.001)$. Sixteen patients $(84.2 \%)$ in group A compared with no patient in group B had normal FAF. The percentages of patients with normal FAF and no disruption in ELM, IS/OS and VM were $78.9 \%$ and $0 \%$ in groups $A$ and $B$, respectively. The difference was statistically significant between the two groups $(p<0.001)$. Visual outcome was significantly affected by the combined effects of ultrastructural changes in the retina and FAF after controlling for preoperative BCVA and macular status $(\mathrm{p}=0.040)$.

Twelve patients (70.6\%) with macular-on RRD and four patients $(20 \%)$ with macular-off RRD had normal FAF. The difference was statistically significant $(p=0.002)$. 
Relationship between microperimetry and BCVA

Seventeen patients underwent MP-1 analysis. Five eyes with unreliable reliability test were excluded. Of the remaining 12 eyes, $11(91.7 \%)$ had stable central fixation, and one (8.3\%) had relatively unstable fixation because of a poor BCVA of $1 / 60$. The mean retinal sensitivities for central $2^{\circ}$ in groups $A$ and $B$ were $15.39 \pm 1.55 \mathrm{~dB}$ and $7.18 \pm 4.74 \mathrm{~dB}$, respectively, while those for the central $20^{\circ}$ were $15.40 \pm 1.17 \mathrm{~dB}$ and $8.76 \pm 4.00 \mathrm{~dB}$, respectively. The Mann-Whitney $U$ test revealed significant differences between the two groups in both $2^{\circ}(\mathrm{p}=0.004)$ and $20^{\circ}$ $(p=0.004)$. Correlations between MP-1 and postoperative BCVA were high in both $2^{\circ}(\rho=-0.747, p=0.005)$ and $20^{\circ}(\rho=-0.778$, $\mathrm{p}=0.003)$.
Correspondence of sdOCT and FAF changes with microperimetry

The areas of decreased sensitivity when mapped onto the FAF images corresponded to areas of ultrastructural changes seen on sdOCT and abnormal FAF (table 3; figure $1 \mathrm{C}, \mathrm{H}, \mathrm{K}$ ). The functional deficits detected by MP-1 corresponded well with abnormal FAF when the FAF and MP-1 images were superimposed and with the structural changes seen on combined sdOCT and FAF images.

Three patients with macular-on RRD developed poor postoperative BCVA. One patient had curvilinear areas of increased FAF (figure 1D,E), one had persistent SRF, and one had a combined ERM and macular fold. These may have contributed to the poor visual outcome observed. Five patients with macularoff RRD had good postoperative BCVA (figure $1 \mathrm{~F}-\mathrm{H}$ ). The

Table 3 Clinical, spectral domain optical coherence tomography and fundus autofluorescence characteristics, and microperimetric sensitivities of patients after repair of rhegmatogenous retinal detachment

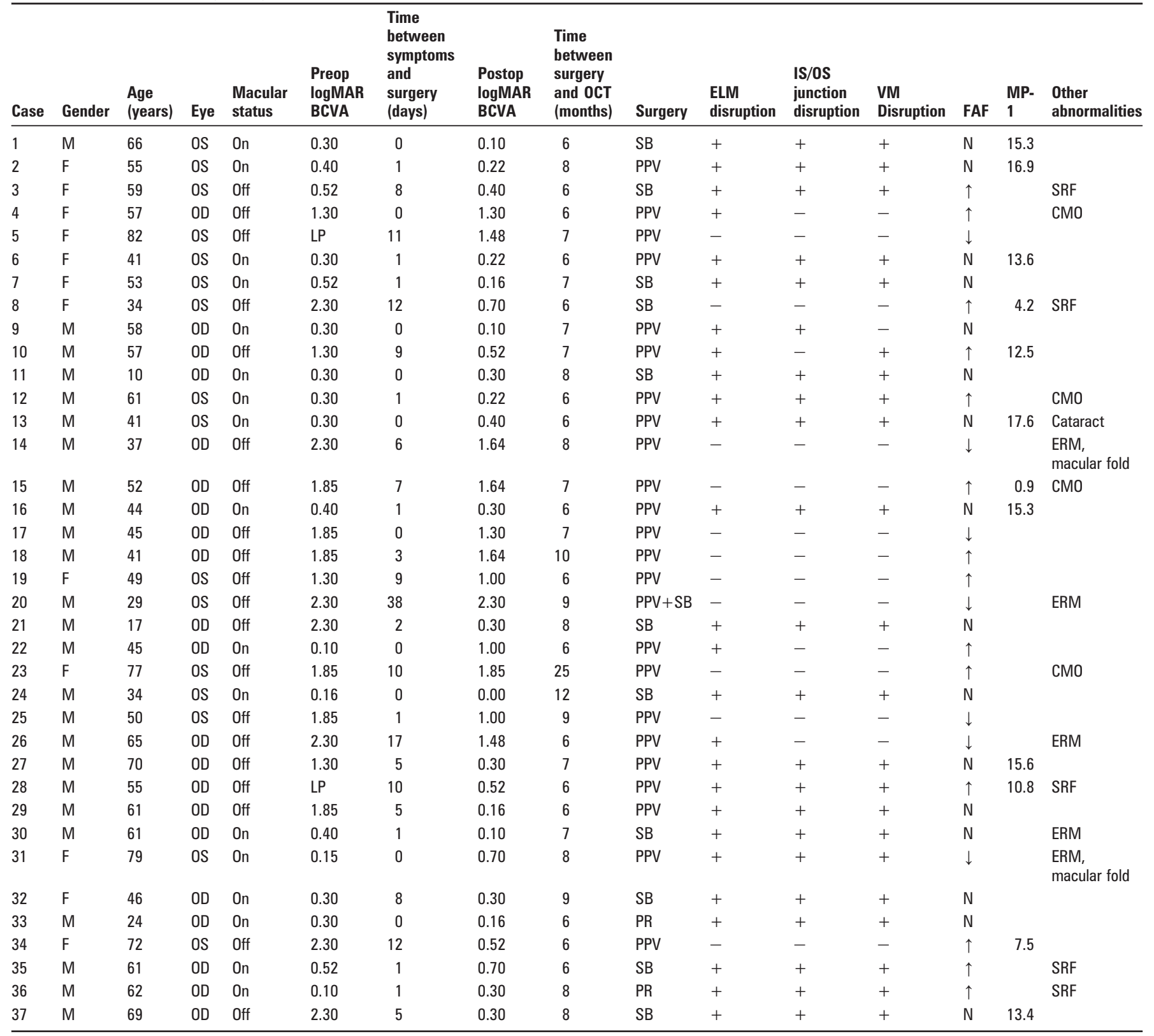

$\uparrow$, hyperfluorescence; $\downarrow$, hypo- or absent fluorescence; CMO, cystoid macular oedema; ELM, external limiting membrane; ERM, epiretinal membrane.; F, female; FAF, fundus autofluorescence; IS/OS, photoreceptor inner and outer segments junction; logMAR BCVA, best-corrected visual acuity in logarithm of minimal angle of resolution; LP, light perception; M, male; MP-1, microperimetry-1; N, normal; OCT, optical coherence tomography; OD, right eye; OS, left eye; Postop, postoperative; PPV, pars plana vitrectomy; PR, pneumatic retinopexy; Preop, preoperative; $\mathrm{SB}$, scleral buckle; SRF, subretinal fluid; VM, Verhoeff membrane; 
average time between the development of symptoms and surgery in these patients was $5.00 \pm 2.12$ days.

\section{DISCUSSION}

Recent advances in imaging technologies have allowed us to detect ultrastructural abnormalities in the macula to histological details and to correlate these changes with functional deficits.
This is the first study that employed simultaneous sdOCT and cSLO imaging, and microperimetry, to examine the ultrastructural and FAF changes and functional deficits after successful repair of $R R D$.

In this study, sdOCT has identified IS/OS disruptions after successful RRD repair. This finding is consistent with those reported previously using time-domain OCT, $^{18}$ ultrahigh
Figure 2 Patient 23. Simultaneous (A) FAF imaging and (B) sdOCT 25 months after RRD repair. A round chorioretinal scar with decreased FAF is noted (arrow). The boundary of the decreased FAF colocalised exactly with the boundary of disruptions of the ELM, IS/ OS and VM on sdOCT (arrowhead and green vertical line). Patient 5. (C) sdOCT and macular thickness map at 3 months after repair of macular-off RRD. There is disruption of the ELM, IS/OS and VM. (D) sdOCT and macular thickness map 15 months after surgery. Partial restoration of the ELM and IS/OS (arrowheads) and an increase in foveal thickness were noted. ELM, external limiting membrane; FAF, fundus autofluorescence; IS/OS, photoreceptor inner and outer segment junction; RRD, rhegmatogenous retinal detachment; sdOCT, spectral domain optical coherence tomography; VM, Verhoeff membrane.
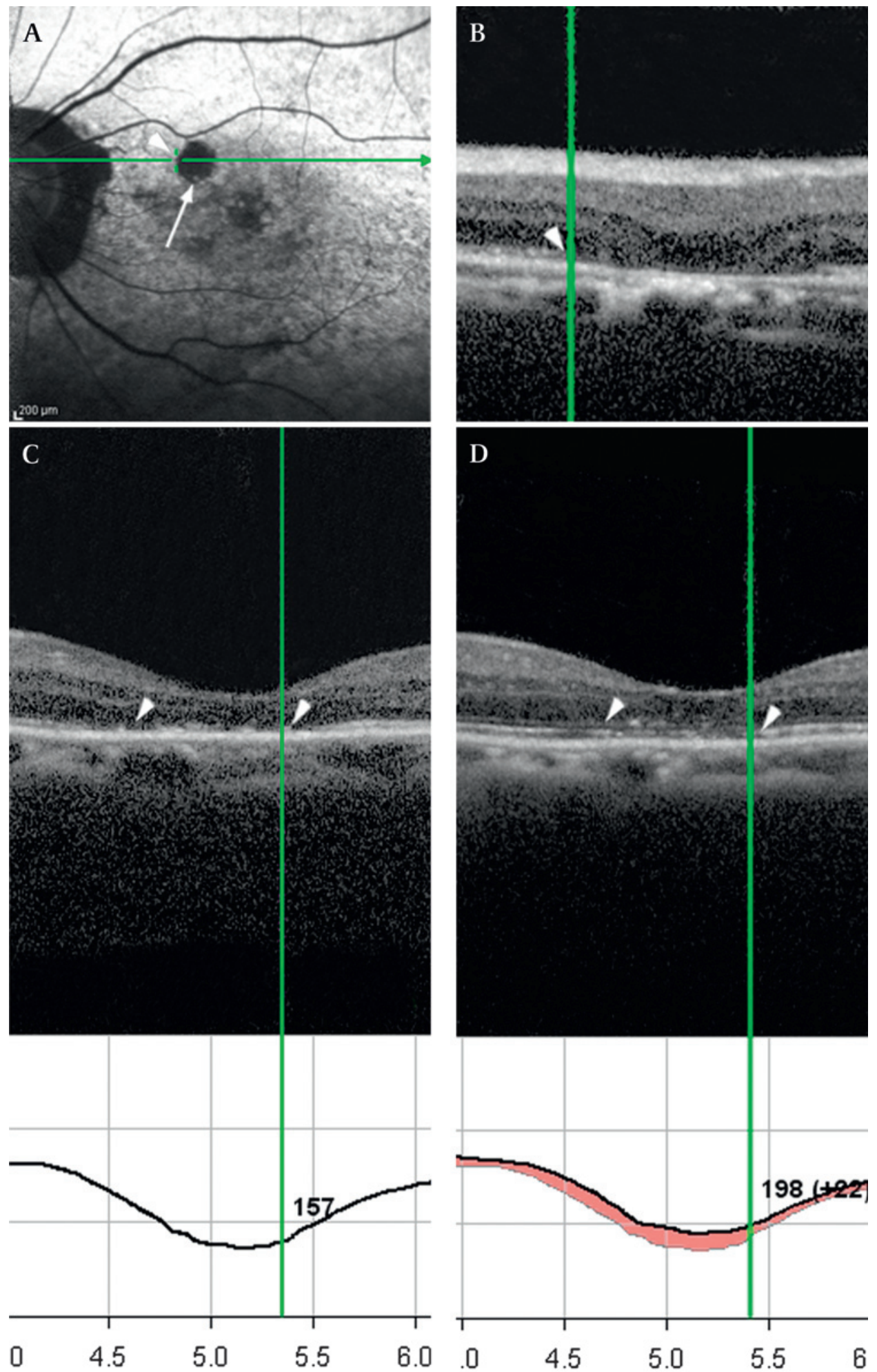

Position [mm] 
resolution ${ }^{6}$ and sdOCT. $^{8}{ }^{10}$ In addition, ELM and VM disruptions were noted in our study. A recent study has observed disruptions of the ELM and IS/OS junction, and found that their combined disruptions portended a poorer visual outcome when compared with those patients with isolated IS/OS disruptions. ${ }^{10}$ In our study, we also noted an additional finding, namely the disruption of VM. This feature appears also to be related to the visual outcome after RRD repair. Statistical analysis shows that the combined effects of ELM, IS/OS and VM disruptions resulted in a significantly poorer visual outcome.

Experimental models have shown that within a few hours after $\mathrm{RRD}$, the apical processes of RPE undergo morphological changes. ${ }^{19}$ Degeneration of the photoreceptor OS follows shortly. With a longer duration of the RRD, the IS becomes disrupted. Apoptosis of photoreceptors has been identified in humans and animals after $\mathrm{RRD}{ }^{20}$ The high-resolution images obtained in our study provided a non-invasive means to visualise outer retinal changes in vivo and to correlate them with reported histopathological findings. Anatomically, the backreflection that arises from the IS/OS junction is formed by the connecting cilia between the IS and OS, while the backreflection that forms the ELM represents zonular adherents which join the IS to the Müller cells. ${ }^{21}$ The backreflection that represents the $\mathrm{VM}^{7}$ is formed by desmosomes on the lateral RPE cell membranes. ${ }^{22}$ Until recently, time-domain ${ }^{18}$ and ultrahigh-resolution $\mathrm{OCT}^{6}$ have only been able to document IS/OS disruptions. In contrast, our study has shown that the disruptions could extend beyond this junction to involve the IS and photoreceptor cell bodies, and the RPE, as evidenced by the disruptions of the ELM and VM, respectively. The results also illustrate that different degrees of disruption may be present in the outer retina despite the clinical impression that the structural relationship between the outer retina and the RPE has been successfully re-established by surgery.

Improper turnover of $O S$ and the displacement of luteal pigments have been implicated as the causes for the increased FAF found in SRF and $\mathrm{CMO}$, respectively. ${ }^{14}$ In our study, we found that the occurrence of ultrastructural disruption of the outer retinal layers was significantly associated with FAF abnormalities. In most patients with ERM or MF, or both, there were severe IS/OS and VM disruptions. The corresponding damage to the RPE cells with loss of lipofuscin may be a cause for the decreased or absent FAF observed. We were, however, surprised that FAF and sdOCT abnormalities did not exactly colocalise in the majority of cases. We speculate that sdOCT detect acute changes, whereas FAF changes may lag behind the ultrastructural changes. ${ }^{13}$ We cannot however confirm this in this study because of its cross-sectional nature and because FAF is not quantifiable. Attempts have been made to quantify FAF in conditions such as age-related macular degeneration, in particular geographic atrophy and Stargardt disease. Such attempts have not been shown to be reliable or reproducible in cases of retinal detachment in which the changes in FAF may be less discrete and more subtle. ${ }^{23}$ Where we have observed exact colocalisation of FAF with sdOCT changes in this series, it occurred in a patient that had retinal detachment repaired more than 2 years previously (figure $2 \mathrm{~A}$ ).

Wakabayashi reported restoration of normal or near-normal retinal architecture, such as the IS/OS junction, after RRD repair. ${ }^{10}$ In this study, we have also seen partial restoration of some of the disrupted layers in one of our patients (figure $2 \mathrm{~B}, \mathrm{C}$ ). This represents a highly intriguing finding. In general, apoptosis, once it has occurred, is regarded as irreversible, and cell division of photoreceptors does not occur. If the architecture could be restored albeit incompletely, this would suggest that not all the observed microstructural changes should be attributed entirely to apoptosis.
Limitations of this study include the relatively small sample size, its retrospective and cross-sectional nature, and the lack of preoperative imaging for comparison. The presence of preoperative maculopathy could not be eliminated, which may influence the postoperative outcomes. Serial imaging was not performed in all our patients to examine the extent of restoration of the outer retinal layers and the changes in FAF over time.

Visual outcome following retinal detachment can often be disappointing despite seemingly good anatomical results. Neuroprotective therapy directed at preventing apoptosis has long been suggested. ${ }^{24}$ The difficulty has been finding objective physical parameters which can be used to measure recovery. In this cross-sectional study, we have shown that ultrastructural abnormalities in the macula can be frequently observed using simultaneous sdOCT and FAF in clinically 'normal' retina and that they corresponded in general to functional deficits as measured by the MP-1 and BCVA. These imaging techniques warrant longitudinal studies to better define the temporal relationship between structural and functional changes, and they may provide the objective parameters needed for a prospective trial on the efficacy of neuroprotection.

Acknowledgements The authors would like to thank the Research Centre of Heart, Brain, Hormone and Healthy Ageing (HBHA), The University of Hong Kong, for their support in this study.

Contributors All authors substantially contributed to the concept, design, analysis and interpretation of data, drafting the article and revising it critically for important intellectual content, and final approval of the version to be published.

Funding The study is supported in part by the University Development Fund, LKS Faculty of Medicine, The University of Hong Kong, Hong Kong.

\section{Competing interests None.}

Ethics approval Ethics approval was provided by the University of Hong Kong/Hospital Authority Hong Kong West Cluster.

Provenance and peer review Not commissioned; externally peer reviewed.

\section{REFERENCES}

1. Tani $\mathbf{P}$, Robertson DM, Langworthy A. Prognosis for central vision and anatomic reattachment in rhegmatogenous retinal detachment with macula detached. Am J Ophthalmol 1981;92:611-20.

2. Wolfensberger TJ, Gonvers M. Optic coherence tomography in the evaluation of incomplete visual acuity recovery after macula-off retinal detachments. Graefe's Arch Clin Exp Ophthalmol 2002;240:85-9.

3. Yetik H, Guzel H, Ozkan S. Structural features of attached retina in rhegmatogenous retinal detachments. Retina 2004;24:63-8.

4. Benson SE, Schlottmann PG, Bunce C, et al. Optical coherence tomography analysis of the macula after vitrectomy surgery for retinal detachment. Ophthalmology 2006;113:1179-83.

5. Hagimura $\mathbf{N}$, Suto K, lida T, et al. Optical coherence tomography of the neurosensory retina in rhegmatogenous retinal detachment. Am J Ophthalmol 2000;129:186-90.

6. Schocket LS, Witkin AJ, Fujimoto JG, et al. Ultrahigh-resolution optical coherence tomography in patients with decreased visual acuity after retinal detachment repair Ophthalmology 2006;113:666-72.

7. Alam S, Zawadzki RJ, Choi S, et al. Clinical application of rapid serial Fourier-domain optical coherence tomography for macular imaging. Ophthalmology 2006;113:1425-31

8. Nakanishi H, Hangai M, Unoki N, et al. Spectral-domain optical coherence tomography imaging of the detached macula in rhegmatogenous retinal detachment. Retina 2009;29:232-42.

9. Smith AJ, Telander DG, Zawadzki RJ, et al. High-resolution Fourier-domain optical coherence tomography and microperimetric findings after macula-off retinal detachment repair. Ophthalmology 2008;115:1923-9.

10. Wakabayashi T, Oshima Y, Fujimoto $\mathrm{H}$, et al. Foveal microstructure and visual acuity after retinal detachment repair: imaging analysis by Fourier-domain optical coherence tomography. Ophthalmology 2009;116:519-28.

11. Delori FC, Dorey CK, Staurenghi G, et al. In vivo fluorescence of the ocular fundus exhibits retinal pigment epithelium lipofuscin characteristics. Invest Ophthalmol Vis Sci 1995; 36:718-29.

12. von Ruckmann A, Fitzke FW, Bird AC. Distribution of fundus autofluorescence with a scanning laser ophthalmoscope. Br J Ophthalmol 1995;79:407-12.

13. Holz FG, Bellman C, Staudt S, et al. Fundus autofluorescence and development of geographic atrophy in age-related macular degeneration. Invest Ophthalmol Vis Sci 2001;:42:1051-56. 
14. Schmitz-Valckenberg S, Holz FG, Bird AC, et al. Fundus autofluorescence imaging. Review and perspectives. Retina 2008;28:385-409.

15. Spaide RF. Autofluorescence from the outer retina and subretinal space. Hypothesis and review. Retina 2008;28:5-35.

16. Wolf-Schnurrbusch UEK, Enzmann V, Brinkmann CK, et al. Morphologic changes in patients with geographic atrophy assessed with a novel spectral OCT-SLO combination. Invest Ophthalmol Vis Sci 2008;49:3095-9.

17. Springer C, Bultmann S, Volcker HE, et al. Fundus perimetry with the Micro Perimeter 1 in normal individuals: comparison with conventional threshold perimetry. Ophthalmology 2005;112:848-54.

18. Lecleire-Collet $\mathbf{A}$, Muraine $\mathrm{M}$, Ménard JF, et al. Evaluation of macular changes before and after successful retinal detachment surgery using Stratus optical coherence tomography. Am J Ophthalmol 2006;142:176-9.
19. Machemer R. Experimental retinal detachment in the owl monkey. II. Histology of retina and pigment epithelium. Am J Ophthalmol 1968;66:396-410.

20. Chang CJ, Lai WW, Edward DP, et al. Apoptotic photoreceptor cell death after traumatic retinal detachment in humans. Arch Ophthalmol 1995;113:880-6.

21. Spitznas M. The fine structure of the so-called outer limiting membrane in the human retina. Albrecht Von Graefes Arch Klin Exp Ophthalmol 1970;180:44-56.

22. Verhoeff FH. A hitherto undescribed membrane of the eye and its significance. Boston Med Surg J 1903;149:456.

23. Sunness JS, Ziegler MD, Applegate CA. Issues in quantifying atrophic macular disease using retinal autofluorescence. Retina 2006;26:666-72.

24. Li SY, Fu ZJ, Ma H, et al. Effect of lutein on retinal neurons and oxidative stress in a model of acute retinal ischemia/reperfusion. Invest Ophthalmol Vis Sci 2009;50:836-43. 